\title{
Buildup of steady-state subpicosecond and femtosecond pulses in a colliding-pulse mode-locked ring dye laser
}

\author{
Jahn-Chung Kuo and Ci-Ling Pan \\ Institute of Electro-optical Engineering, Center for Telecommunication Research, National Chiao Tung University, Hsinchu, \\ Taiwan 30050, China
}

Received April 18, 1990; accepted September 13, 1990

\begin{abstract}
We show for the first time, to our knowledge, that the buildup time to steady-state femtosecond pulses in a typical colliding-pulse mode-locked dye laser $(\lambda=620 \mathrm{~nm})$ with compensating prisms is $15 \mu \mathrm{sec}$ after the pulses have been completely modulated. For a similar laser without the compensating prisms, the buildup time is $40 \mu \mathrm{sec}$ and exhibits interesting oscillatory behavior in the initial stage.
\end{abstract}

Since it was first demonstrated by Fork et al., ${ }^{1}$ the colliding-pulse mode-locked (CPM) dye laser, which can produce stable subpicosecond to femtosecond pulses, has been extensively studied both experimentally ${ }^{1-3}$ and theoretically. ${ }^{4-8}$ Without considering the phase-modulation (chirping) effects, it was generally believed that steady-state energy and pulse durations were reached in a few microseconds, corresponding to tens of to a few hundred round trips. ${ }^{4-7}$ Earlier experimental research on the flash-lamp-pumped passively mode-locked Rhodamine 6G/DODCI dye laser showed that the laser output quickly evolved into 2psec pulses after $\sim 35$ round trips, ${ }^{9}$ while such a laser operated in the CPM configuration evolved from noise bursts to single pulses of $<1.5$ psec after $\sim 150$ round trips. ${ }^{10}$ It was thought that the higher loss and longer length of the cavity used were largely responsible for the increase in the buildup time. Recently, Ansari et $a l .{ }^{11}$ stated that an average mode-locked pulse width of $0.34 \mathrm{psec}$ in the pulse train appeared at $\sim 20 \mu \mathrm{sec}$ after the start of the dye-laser output in their novel CPM dye laser pumped by a pulsed xenon-ion laser. This was attributed primarily to the rise and overshoot of the pump pulse, while the subpicosecond pulse train was thought to develop in a fraction of a microsecond. The effects of self-phase modulation and group-velocity dispersion, however, have not been considered in these studies. Since both group-velocity dispersion and self-phase modulation broaden the pulse width, these effects are expected to prolong the buildup time of subpicosecond pulses reaching the steady state. By compensating the chirping, the pulses should evolve to the steady state more rapidly. In this Letter we report for the first time, to our knowledge, results on the evolution of the pulse widths and the corresponding spectra of subpicosecond and femtosecond pulses generated by a CPM dye laser to the steady state with and without the compensating prism sequence.

A block diagram of our experimental setup is shown in Fig. 1. The CPM dye-laser system was of the standard seven-mirror configuration. The cavity roundtrip time was 10 nsec. All the cavity mirrors were single-stack dielectric coated with the center wavelength at $616 \mathrm{~nm}$ and a $100-\mathrm{nm}$ bandwidth. The dye concentrations were $2 \times 10^{-3}$ and $3 \times 10^{-4} \mathrm{M}$ for the gain (Rhodamine 590) and absorber (DODCI) dyes, respectively. The dye laser was pumped by a cw argon-ion laser at 3.2 and $1.8 \mathrm{~W}(\lambda=514.5 \mathrm{~nm})$ for the system with and without the compensating prism sequence, respectively. The lasing threshold for the CPM laser is less than $2.5 \mathrm{~W}$ in the former case and $\sim 1.3 \mathrm{~W}$ in the latter case. In order to investigate the buildup behavior of the CPM dye laser, the cw argonion pump laser was mechanically chopped to obtain $800-\mathrm{Hz}$ square pulses with a FWHM of $500 \mu \mathrm{sec}$ and a rise time of $30 \mu \mathrm{sec}$. Line traces of the typical pump pulse and the corresponding output of the dye laser are also shown in Fig. 1. The CPM laser output was fed to a noncollinear autocorrelator or a monochrometer and detected by a gatable photomultiplier tube (PMT) with a 50-nsec gate to select five pulses. A boxcar integrator with a 100-nsec gate was used for

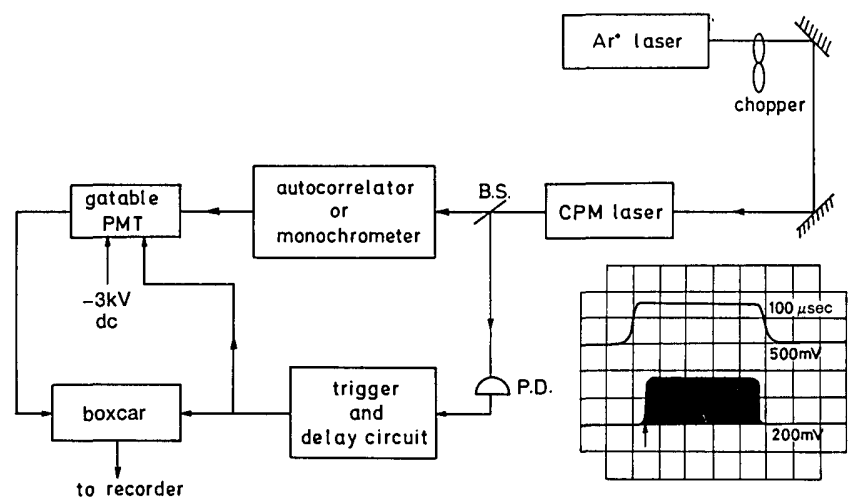

Fig. 1. Block diagram of experimental arrangement. B.S., beam splitter; P.D., photodiode. In the window, the upper trace is the chopped argon-ion laser pump pulse with a FWHM of $500 \mu \mathrm{sec}$ and a rise time of $30 \mu \mathrm{sec}$, and the lower trace is the CPM dye-laser output. The arrow marks the zero delay time, which is chosen to be the instant corresponding to a $10 \%$ rise in the dye-laser output. The horizontal scale is $100 \mu \mathrm{sec} /$ division. 


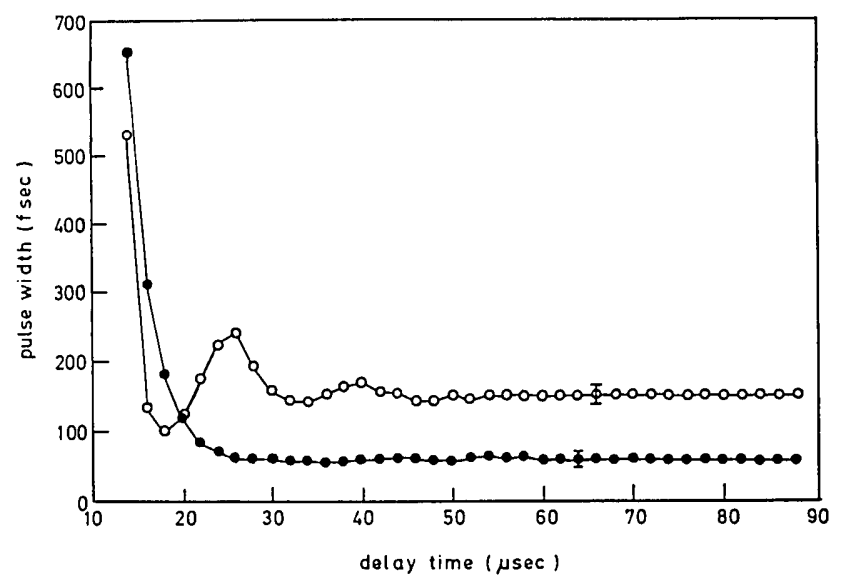

Fig. 2. Pulse width as a function of the delay time from the onset of laser action for the CPM laser with (close circles) and without (open circles) the compensating prism sequence.

signal averaging. By delaying these two synchronous gates with respect to the onset of dye-laser action, we can sample the evolution of temporal and spectral characteristics of the CPM laser pulses.

Figure 2 illustrates the laser pulse width as a function of the delay time for the CPM laser with and without the compensating prism sequence. A sech ${ }^{2}$ pulse shape is assumed. The pulse energies also exhibited a similar trend. Zero delay time corresponded to the onset of dye-laser action. Initially the incompletely modulated pulses built up from noise. As these pulses circulated inside the cavity and interacted with the gain and absorber media, their energies built up and the pulses became completely modulated with a pulse width of a few picoseconds in $\sim 10 \mu$ sec. This initial buildup time depends on the rise time, the pulse shape, and the peak power of the pump laser. ${ }^{11}$ In our case it was due to the slow rise time $(\sim 30 \mu \mathrm{sec})$ of the mechanically chopped argon-ion pump laser. With saturated gain and absorption, the pulses narrowed to subpicosecond pulses quickly in a few microseconds. The pulse width approached steady state in $\sim 25 \mu$ sec. Excluding the initial mode-locking regime, the buildup time to steady state took $15 \mu \mathrm{sec}$. This is consistent with the theoretical results given by Petrov et al. ${ }^{6}$ based on numerical calculations taking the pulse modulation effects into account. The corresponding spectra are shown in Fig. 3. The peak wavelength shifted slightly to the red as the pulses evolved, but otherwise the spectra did not exhibit distinct features. Without the prism sequence to compensate intracavity chirp, the initial buildup time for the formation of completely modulated pulses was essentially the same as in the previous case, i.e., $\sim 10 \mu \mathrm{sec}$. The pulse width exhibited an oscillatory behavior and approached the steady state after a time delay of $\sim 40$ $\mu$ sec, excluding the initial buildup time for completely modulated pulses. The corresponding spectra of the laser output are given in Fig. 4. A dramatically changed spectrum was also observed at the first minimum of oscillation. The oscillation is consistent with the competition between self-phase modulation and group-velocity dispersion with the conventional mode-locking mechanisms, i.e., the saturation of the gain and absorber. As the intracavity pulse energy increased, while the pulses narrowed initially owing to saturable gain and absorption, the self-phase modulation due to the dominant fast Kerr effect in the dye solvent for our system increased at the same time. This broadened the spectrum of the pulses, and the accompanying group-velocity dispersion broadened the pulse width. This in turn decreased the intracavity peak intensity of the pulses, and the self-phase modulation and group-velocity dispersion decreased as a result. In the meantime, the pulses became narrower. This process repeated itself until the pulses reached the steady state. As a result, the buildup time to steady state was longer than in the system with the compensating prism sequence. At the initial stage of pulse development (delay time $<20 \mu \mathrm{sec}$ ), since the pulse durations were longer and the phase-modulation effect had not built up, the additional prism sequence contributed an uncompensated group-velocity dispersion and was responsible for the longer pulse width observed for the system with the prisms compared with that of the system without the prism sequence.

Finally, we discuss briefly the influence of the pump power on the buildup behavior of the CPM dye laser. Jacobovitz et $a .^{12}$ showed that, in the steady state, there is a pronounced minimum pulse duration by varying the pump power and optimizing the absorber concentration. At higher intracavity energies, the pulse widths broadened owing to the increase of the chirp. ${ }^{13}$ It is thus expected that at a higher pump power, the entire buildup curve for the system without

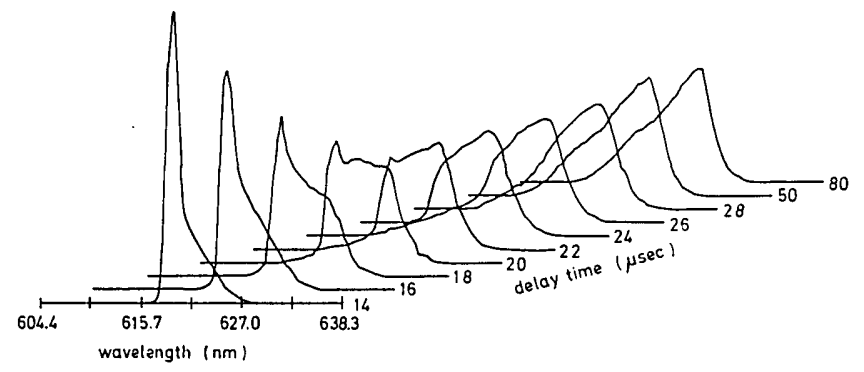

Fig. 3. Spectra of the CPM laser with the compensating prism sequence as a function of delay time.

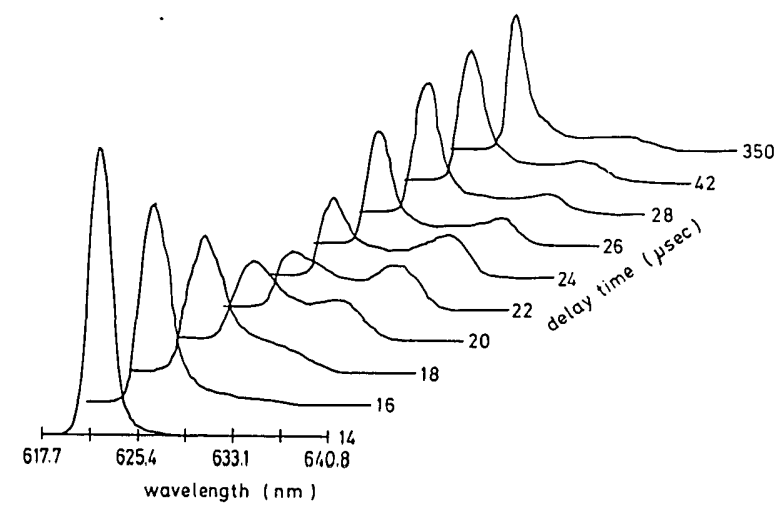

Fig. 4. Spectra of the CPM laser without the compensating prism sequence as a function of delay time. 
the compensating prisms should be elevated, with the steady-state pulse width broadened. In contrast, the buildup behavior of a dispersion-compensated CPM laser should be insensitive to the pump power dependence with appropriate adjustment of the prisms at different pump powers. Increasing the pump power should also shorten the initial buildup time for the formation of completely modulated pulses. ${ }^{11}$

In summary, we have presented detailed data on the evolution of subpicosecond to femtosecond pulses and their spectra generated by a CPM dye-laser system. Interesting oscillatory behavior in the pulse width was observed, and it is explained by the combined action of saturable absorption, saturable gain, self-phase modulation, and group-velocity dispersion. With the compensating prism sequence used to minimize the pulse chirp, the buildup time to the steady state is approximately $15 \mu \mathrm{sec}$, excluding the initial stage of formation of completely modulated pulses for our laser system. This is nearly three times shorter than with a laser without the compensating prisms.

This research was partially supported by the $\mathrm{Na}$ tional Science Council of the Republic of China under grant NSC78-0608-E009-06.

\section{References}

1. R. L. Fork, B. I. Greene, and C. V. Shank, Appl. Phys. Lett. 38, 671 (1981).

2. W. Dietel, J. J. Fontaine, and J. C. Diels, Opt. Lett. 8, 4 (1983).

3. J. A. Valdmanis and R. L. Fork, IEEE J. Quantum Electron. QE-22, 112 (1986).

4. K. K. Li, G. Arjavalingam, A. Dienes, and J. R. Whinnery, IEEE J. Quantum Electron. QE-19, 539 (1983).

5. M. Yoshizawa and T. Kobayashi, IEEE J. Quantum Electron. QE-20, 797 (1984).

6. V. Petrov, W. Rudolph, and B. Wilhelmi, Opt. Quantum Electron. 19, 377 (1987).

7. M. S. Stix and E. P. Ippen, IEEE J. Quantum Electron. QE-19, 520 (1983).

8. O. E. Martinez, R. L. Fork, and J. P. Gordon, Opt. Lett. 9, 156 (1984).

9. R. S. Adrain, $\mathrm{Ph} . \mathrm{D}$. dissertation (The Queen's University of Belfast, Belfast, UK, 1974).

10. S. Singh, Appl. Opt. 26, 66 (1987).

11. H. Ansari, A. Dienes, and J. R. Whinnery, Opt. Lett. 10, 19 (1985).

12. G. R. Jacobovitz, C. H. Brito Cruz, and M. A. Scarparo, Opt. Commun. 57, 133 (1986).

13. R. S. Miranda, G. R. Jacobovitz, C. H. Brito Cruz, and M. A. F. Scarparo, Opt. Lett. 11, 224 (1986). 